\section{Vesicle-based artificial cells: recent developments and prospects for drug delivery}

\begin{abstract}
"...the major driving principle behind research in this area aims to address the following question: can we mimic and harness the power of biology to create new classes of smart micromachines that can be engineered for functional purposes?"
\end{abstract}

Keywords: artificial cells • biomimicry • drug delivery • microfluidics • micromachines - synthetic biology $\bullet$ vesicles

Over evolutionary history cells have developed a range of features which are now considered essential for life, including replication, compartmentalization, metabolism, movement, communication and environmental sensing and subsequent response. Recent technological developments have meant that these features have started to find their way into artificially constructed, nonbiological structures. These are commonly known as artificial cells, but are also referred to in the literature as protocells, minimal cells or synthetic cells. Usually constructed from the bottom up, artificial cells often hijack biological machinery (DNA, enzymes, protein pores) - either natural or engineered - to perform designated functions. Beyond simple scientific curiosity the major driving principle behind research in this area aims to address the following question: can we mimic and harness the power of biology to create new classes of smart micromachines that can be engineered for functional purposes?

Although the field is still in its relative infancy substantial inroads have been made toward this vision. What is already clear is that prospects for this technology are considerable, ranging from environmental sensing of toxins to medicinal contrast imaging. However, the greatest potential applications lie in the realm of therapeutics: for responsive in vivo drug synthesis, engineered communication with diseased cells, hosting genes for gene therapy, and delivery of therapeutic enzymes, peptides and transplant cells. The scenario can be envisaged in which an arti- ficial cell monitors its surroundings, moves toward its target site and initiates on-site drug synthesis and subsequent secretion upon arrival. The introduction of such biomimetic features will represent a step change in complexity from current drug delivery paradigms, and could prove a disruptive innovation for the therapeutic industry.

The most common chassis that define the boundaries of artificial cells are phospholipid vesicles. The reasons for this stem from their similarity to biological membranes in their morphology (enclosed spherical bilayer shells), size regime (micron or submicron diameters) and chemistry (composed of cellular lipids or their analogs). Crucially, the use of biological building blocks enhances biocompatibility and allows natural degradation pathways to be used. Furthermore, the rich phase behavior that lipids display - different phases possessing different degrees of order, viscosities and permeabilities - can be utilized for the introduction of responsiveness, as phase transitions can be induced by the external environment. Finally, using vesicles enables biological machinery to be embedded in the membrane, in particular transmembrane pores and receptors.

The well-established use of vesicles in therapeutics has seen them acting as capsules for the delivery of drugs, both hydrophobic (embedded in the bilayer core) and hydrophilic (encapsulated in the inner vesicle volume) [1]. Importantly, vesicles have been shown to offer the potential to selectively target diseased cells [2]. In terms of generating

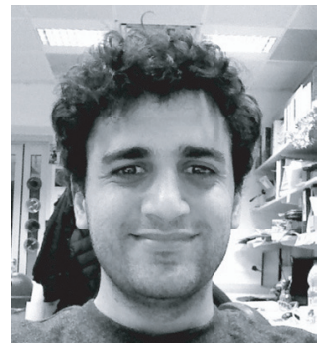

Yuval Elani

Department of Chemistry, Imperial College London, Exhibition Road, London, SW7 2AZ, UK

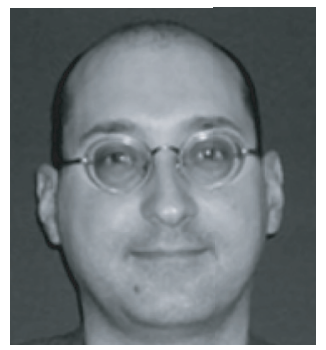

Robert V Law

Department of Chemistry, Imperial College London, Exhibition Road, London, SW7 2AZ, UK

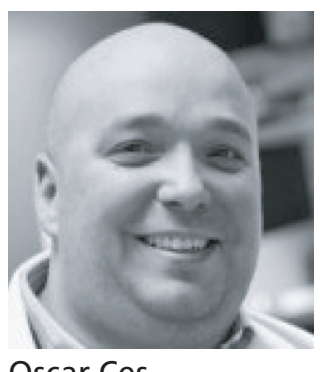

Oscar Ces

Author for correspondence: Department of Chemistry, Imperial College London, Exhibition Road, London, SW7 2AZ, UK o.ces@imperial.ac.uk

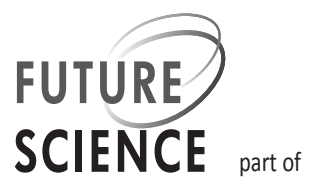
fsg 
vesicle-based artificial cells with properties that may be useful in a drug-delivery context, there have been some striking recent achievements. The cells have acted as microreactors for the synthesis of small organic compounds [3]. They have been functionalized to grow, replicate and move [4]. They have been shown to communicate with surrounding cells and to participate with their quorum-sensing mechanisms [5]. Within these cells DNA amplification [6] and in vitro protein synthesis has taken place, with continual protein production due to the uptake of biochemical feed-stocks from the surrounding environment [7].

One of the key milestones has been the introduction of compartmentalization: the splitting of a vesicle's internal volume into discrete regions [8-10]. This enables modular multipart delivery systems to be constructed, with each compartment existing in distinct biochemical environments, and optimized to perform specific tasks in a manner analogous to eukaryotic organelles. Notably, chemical cross-talk between compartments has also been shown, demonstrating their use as a platform with which benchtop chemistry can be performed in the interior of vesicles [3]. These developments will help facilitate the incorporation of emergent features into vesicle-based artificial cells.

\section{"One of the key milestones has been the introduction of compartmentalization: the splitting of a vesicle's internal volume into discrete regions."}

Importantly in a therapeutic context, compartmentalization allows spatial organization in artificial cells, offering the potential for dormant systems to be constructed where inactive drug precursors are isolated from one another and only activated when appropriate stimuli are encountered. The resulting in vivo drug synthesis could prove to be an immensely useful strategy to widen a pharmaceutical's therapeutic window, and for smart cells to be administered prophylactically, lying dormant in the body only becoming activated in the event of future illness.

It is also important to recognize that in addition to the incorporation of embedded natural or engineered proteins, there are other types of machinery that could be utilized, including DNA origami (defined 2D and 3D DNA architectures) [11] as well as nanoparticles (quantum dots and polymeric capsules) [12,13].

In order to predict future directions and challenges of the field, it is instructive to consider the reasons for its recent emergence and rapid growth in the first place. It can be partly attributed to recent innovations relating to vesicle generation strategies [14]. These have largely been droplet-based methods, and often have microfluidic technologies at their core [15]. This enables fine control over vesicle morphology, content and size, and allows scale-up in generation throughput. Such technologies have for the first time made it possible to easily and reliably encapsulate a range of large, charged biological molecules within them - a prerequisite for the construction of artificial cells - and have enabled the construction of interconnected networks of hundreds of cell-like units [16]. In addition, the commercialization of in vitro biological components and the ever-decreasing cost of DNA synthesis and sequencing has also made it easier and cheaper to introduce biological elements into vesicles [17].

Further growth of the field and the realization of its wider potential will depend on effective synergies with related disciplines, in particular with top-down synthetic biology, where engineering principles and classical molecular biology techniques are used to construct biological circuits and devices [18]. By incorporating these into vesicle-based artificial cells, increasingly sophisticated functionality can be achieved with many potential applications in medicine. In the simplest example, by coupling appropriate inducers and repressors, synthesis of therapeutic proteins could be activated on-site in response to relevant stimuli [19]. More complex scenarios would involve oscillating circuits and logic gates that could lead to higher-order behaviors. Substantial investments are currently being made in synthetic biology technologies, and the integration of these with vesicle-based artificial cells could be particularly rewarding. In addition, engagement with the chemical biology, membrane-biophysics, nanotechnology and pharmacology communities is needed for the fulfillment of the ambitions of the field of vesicle-based artificial cells.

Despite recent achievements, a clear-headed view of the challenges is needed. First, the cell-like features incorporated into vesicles have often been developed in isolation, with the cells mostly performing a single function - it will be necessary to combine many of these features together for functional use and where possible do so with a view to generating emergent behavior. Secondly, the types of chemicals that can be synthetized into vesicles have largely been restricted to biological biopolymers: RNA, DNA and proteins. Organic compounds are more difficult to synthesize due to their increased permeability to lipid membranes.

The third challenge relates to economic viability: although high-throughput microfluidic techniques offer greater potential for scale up, the cost of the starting reagents is still too high for large-scale commercial use. However, as demonstrated by the exponential yearly decrease in price of DNA synthesis and sequencing (the latter decreasing by a factor of 10,000 in the 
past 6 years), this difficulty is likely to be resolved in the near future if the necessary investment is made.

Fourth, some of the downstream criteria for the use of artificial cells in medicine need to be proven: they should be small and robust enough to pass through capillaries; they need to be effectively cleared from circulation after use; they should not be degraded by the immune response and they should not interfere with the regular functions of cells (except where explicitly designed to do so). The final hurdle that needs to be overcome is a societal one - to convince stakeholders, including members of the public, that the ethical and safety concerns often associated with these technologies have been properly addressed by the scientific community [20].

The use of artificial cells in medicine is perhaps still decades away, but it is clear that the potential is already present. This is a rapidly developing field and one which, interestingly, has emerged in the same era as that of the maker movement where DIY culture has been extended into the realms of technology and prototype development. As a result, synthetic biology and

\section{References}

1 Samad A, Sultana Y, Aqil M. Liposomal drug delivery systems: an update review. Curr. Drug Deliv. 4(4), 297-305 (2007).

2 Fenske DB, Chonn A, Cullis PR. Liposomal nanomedicines: an emerging field. Toxicol. Pathol. 36(1), 21-29 (2008).

3 Elani Y, Law RV, Ces O. Vesicle-based artificial cells as chemical microreactors with spatially segregated reaction pathways. Nat. Commun. 5, 5305 (2014).

4 Kurihara K, Tamura M, Shohda K-I, Toyota T, Suzuki K, Sugawara T. Self-reproduction of supramolecular giant vesicles combined with the amplification of encapsulated DNA. Nat. Chem. 3(10), 775-781 (2011).

5 Gardner PM, Winzer K, Davis BG. Sugar synthesis in a protocellular model leads to a cell signalling response in bacteria. Nat. Chem. 1(5), 377-383 (2009).

6 Lee $\mathrm{S}, \mathrm{Koo} \mathrm{H}, \mathrm{Na} \mathrm{JH}$ et al. DNA amplification in neutral liposomes for safe and efficient gene delivery. ACS Nano 8(5), 4257-4267 (2014).

7 Noireaux V, Libchaber A. A vesicle bioreactor as a step toward an artificial cell assembly. Proc. Natl Acad. Sci. USA 101(51), 17669-17674 (2004).

8 Elani Y, Gee A, Law RV, Ces O. Engineering multicompartment vesicle networks. Chem. Sci. 4(8), 3332-3338 (2013).

9 De Hoog H-PM, Nallani M, Tomczak N. Self-assembled architectures with multiple aqueous compartments. Soft Matter 8(17), 4552-4561 (2012).

10 Beales PA, Vanderlick TK. Application of nucleic acidlipid conjugates for the programmable organisation artificial cell clubs are emerging around the world in community-led Hackspaces. This means that, in the future, innovation in this field will not only occur in university laboratories but also the garages of budding bioentrepreneurs who will benefit from the deskilled technologies that are revolutionizing the life sciences.

\section{Financial \& competing interests disclosure}

This work was supported by the EPSRC via grants EP/ J017566/1, EP/K038648/1, EP/G00465X/1, and by an EPSRC Doctoral Prize Fellowship awarded to $Y$ Elani. The authors have no other relevant affiliations or financial involvement with any organization or entity with a financial interest in or financial conflict with the subject matter or materials discussed in the manuscript apart from those disclosed.

No writing assistance was utilized in the production of this manuscript.

\section{Open Access}

This work is licensed under the Creative Commons Attribution 4.0 License. To view a copy of this license, visit http://creativecommons.org/licenses/by/4.0/

of liposomal modules. Adv. Colloid Interface Sci. 207, 290-305 (2014).

11 Langecker M, Arnaut V, Martin TG et al. Synthetic lipid membrane channels formed by designed DNA nanostructures. Science 338(6109), 932-936 (2012).

12 Chen C-S, Yao J, Durst RA. Liposome encapsulation of fluorescent nanoparticles: quantum dots and silica nanoparticles. J. Nanopart. Res. 8(6), 1033-1038 (2006).

13 Mufamadi MS, Pillay V, Choonara YE et al. A review on composite liposomal technologies for specialized drug delivery. J. Drug. Deliv. 2011, 939851 (2011).

14 Walde P, Cosentino K, Engel H, Stano P. Giant vesicles: preparations and applications. ChemBioChem 11(7), 848-865 (2010).

15 Van Swaay D. Microfluidic methods for forming liposomes. Lab Chip 13(5), 752-767 (2013).

16 Elani Y, Demello A, Niu X, Ces O. Novel technologies for the formation of 2-D and 3-D droplet interface bilayer networks. Lab Chip 12(18), 3514-3520 (2012).

17 Carlson ED, Gan R, Hodgman CE, Jewett MC. Cell-free protein synthesis: applications come of age. Biotechnol. Adv. 30(5), 1185-1194 (2012).

18 Khalil AS, Collins JJ. Synthetic biology: applications come of age. Nat. Rev. Genet. 11(5), 367-379 (2010).

19 Miller D, Booth PJ, Seddon JM et al. Protocell design through modular compartmentalization. J. R. Soc. Interface 10(87), 20130496 (2013).

20 Bedau M, Parke EC. The Ethics of Protocells: Moral and Social Implications of Creating Life in the Laboratory. The MIT Press, Cambridge, MA, USA (2009). 